\title{
Predefined Exponential Basis Set for Half-Bounded Multi Domain Spectral Method
}

\author{
Fahhad Alharbi \\ King Abdulaziz City for Science and Technology, Riyadh, Saudi Arabia \\ E-mail: alharbif@kacst.edu.sa \\ Received March 25, 2010; revised June 29, 2010; accepted July 2, 2010
}

\begin{abstract}
A non-orthogonal predefined exponential basis set is used to handle half-bounded domains in multi domain spectral method (MDSM). This approach works extremely well for real-valued semi-infinite differential problems. It spans simultaneously wide range of exponential decay rates with multi scaling and does not suffer from zero crossing. These two conditions are necessary for many physical problems. For comparison, the method is used to solve different problems and compared with analytical and published results. The comparison exhibits the strengths and accuracy of the presented basis set.
\end{abstract}

Keywords: Multi-Domain Spectral Method, Meshfree Numerical Method, Non-Orthogonal Predefined, Exponential Basis Set, Half-Bounded Domain, Exponential Decay, Quantum Wells, Optical Waveguide

\section{Introduction}

With the growing complexities of the numerically studied problems in natural and applied sciences, spectral method (SM) starts gaining more attention mainly because of its high level of analyticity. This is resulted from its meshfree nature, which reduces the computational memory and time requirements where a major part of the problem is solved analytically. Different spectral methods have been developed since 1970s mainly from applied mathematical perspective. Despite of their superior mathematical performance, applying them was very limited when compared to finite difference (FDM) and finite element methods (FEM).

As known, SM is a special family of the weighted residual methods where the unknown functions are approximated by either an expansion of or interpolation using preselected basis sets. In this paper, the expansion method is used. Spectral methods work very well for homogeneous and smooth computational windows. But, it suffers from the Gibbs phenomenon if the structural function of the studied problem is not analytical. The Gibbs phenomenon is generally a peculiarity of any functional approximation at simple discontinuity. To avoid this problem, multi domain spectral method (MDSM) is developed where the computational window is divided into homogeneous domains where the discontinuities lie at the boundaries. Then, the spectral method is applied in each domain alone to build the matrices and vectors. These are then joined by applying the proper boundary conditions between domains [1-4].

\section{The Exponential Basis Set}

In many real-valued physical system, the extensions toward infinities decay exponentially as:

$$
f \propto e^{ \pm \alpha x}
$$

where \pm is used to cover both $\mp \infty$ with positive $\alpha$. In SM and MDSM, this is one of the main problems [5-9]. A review paper by Shen and Wang discusses this in further details [10]. To overcome this problem, many techniques where used. They can be classified in the following three main categories:

1) Using exponentially decaying functions such as physical Hermite and Laguerre functions and rational Chebyshev and Legendre polynomials. Some other basis are used as well. Physical Hermite and Laguerre functions are decaying exponentially toward infinities as $e^{-x^{2} / 2}$ and $e^{-x / 2}$ respectively. This predefines a narrow ranged decay rate and hence limit the generality. They were adopted in studying phenomena that were known that they can be analyzed using such functions. For example, Laguerre function is the base for radial extension of 
electron wave functions in hydrogenic atoms. So, it is expected to work for electronic distributions of some hydrogenic like atoms.

In rational Chebyshev and Legendre polynomials, the coordinates are transformed rationally to map $(-\infty, \infty)$ or $[0, \infty)$ into $[-1,1]$. Other forms of coordinate transformation were used as well. However, in general, this method suffers from the narrow ranged predefined decay rate. Also, mapping infinite intervals into small finite ones adds often some complexity and approximation errors.

Beside the narrow ranged predefined decay rate and because a finite number of bases can be used practically, this approach inherently forces many zero crossing since most of the used bases are forms of Jacobi polynomials which has $N$ zeros for the $N^{\text {th }}$ order polynomial. In many physical problems, this is expected and allowed. However, in physical system where the decay is behaving as described by Equation (1), this should not be the case.

2) Truncating the numerical window; this is used widely as well. The unbounded window is truncated and additional boundary conditions are used to force an asymptotic exponential behavior, i.e., the function and its first derivative vanish at the truncating points. This reduces the analytical accuracy of SM by adding the truncation error. Also, this does not eliminate zero crossing and hence it doesn't fit the system with Equation (1) exponential decay.

3) Single scaling of the coordinates; this is similar to the first category; but with coordinate scaling where

$$
x \Rightarrow c x
$$

Therefore the predefined decay rate is also scaled. The scaling factor $c$ is chosen intuitively to fit the studied system. However, this results in losing the generality and missing many eigen solutions in eigenvalue problems where the decaying rates for the different eigenvalue solutions are generally different.

The presented method overcomes zero-crossing and single scaling problems by approximating the decaying domain functions by exponential basis set which spans wide range of decaying rates as follows:

$$
f=\sum_{n=0}^{N} a_{n} u_{n}=\sum_{n=0}^{N} a_{n} e^{-\alpha_{n} x}
$$

where

$$
\alpha_{n}=b^{d_{s}+\frac{n}{N}\left(d_{e}-d_{s}\right)}
$$

$b$ is the used exponential base and $d_{s}$ and $d_{e}$ are the smallest and largest used powers respectively. They should be predefined intuitively based on the studied problem. But, they allow many possible decay rates with very small number of bases. For example, by setting $N=$
$10, b=10, d_{s}=-5$, and $d_{e}=5,11$ basis can be used to approximate any exponential function with decay rates between 0.00001 and 100000 .

This basis set is then used directly within the frame of MDSM where Tao method is used for boundary conditions $[1,4]$. Since the base functions are only exponentials, differentiation and integration can be handled easily and analytically. For detailed implementation of MDSM, we would refer the readers to $[1,4]$ for the details. However, listed below are some of the main implementation issues of the presented basis set within the frame of MDSM.

\subsection{Coordinate Transformation}

The selected bases form (Eg. 3) assumes that $x \in[\kappa, \infty)$. For simplicity, $\kappa$ is set to 0 . As standardly done in MDSM, coordinate transformation is used to extend the range of the selected basis set. The presented basis set can be used only for domains that extended into $\pm \infty$. So, the following coordinate transformations can be used.

1) For domains where $x \in[v, \infty)$,

$$
x \Rightarrow x^{\prime}+v
$$

2) For domains where $x \in(-\infty, \omega]$,

$$
x \Rightarrow x^{\prime}+\omega
$$

\subsection{Scalar Products}

As known, MDSM is highly associated with calculating the scalar products $\left(u_{m}|H| u_{n}\right)$. Hereunder listed are some of the common scalar products that are used in MDSM:

1) if $H$ is a multiplication by a constant $d$, then:

$$
\left(u_{m}|d| u_{n}\right)=\frac{d}{\alpha_{n}+\alpha_{m}}
$$

2) if $H$ is a multiplication by $x^{\prime k}$, then:

$$
\left(u_{m}\left|x^{\prime k}\right| u_{n}\right)=\frac{k !}{\left(\alpha_{n}+\alpha_{m}\right)^{k+1}}
$$

3) if $H$ is $k^{\text {th }}$ derivative, then:

$$
\left(u_{m}\left|\frac{d^{k}}{d x^{\prime k}}\right| u_{n}\right)=\frac{\left(-\alpha_{n}\right)^{k}}{\alpha_{n}+\alpha_{m}}
$$

\section{Comparisons}

The presented set was used to analyze successively many optical and quantum electronics. To illustrate its accuracy and validity, two application examples are shown. 
The first one is to approximate a wide range of exponentially decaying functions using the same basis sets, while the second is to obtain the quantized energy states in semiconductor quantum well (QW).

\subsection{Approximating Wide Range of Exponentially Decaying Functions}

In this subsection, the presented set is applied to approximate five exponentially decaying functions and is compared with an approximation using physical Laguerre basis set. The used decaying rates are $0.00535,0.0632$, $0.752,81.2$, and 926. These were picked randomly. Figure 1 shows the obtained approximation using an unscaled physical Laguerre basis set. It is clear that with 25 physical Laguerre functions, only two functions out of the five are approximated adequately. Yet, the method is converging but rather slowly for the remaining functions. For $e^{-0.752 x}$, very few bases are needed to approximate the function to an adequate accuracy. If scaling was used, only two or mostly three functions would be approximated adequately depending on the scaling. For many eigenvalue problems, this is a very serious limitation where different eigenvalues have difierent decaying rates. So, only a small range of the eigenvalues can be obtained accurately.

The same five functions are approximated using the presented exponential basis set with $b=10, d_{s}=-4$, and $d_{e}=4$. The resulted approximations and their associated errors are shown in Figure 2. All the five functions were approximated adequately using the same exponential basis set and the convergence is geometric as can been seen. In many applications, it is crucial to find wide range of eigenvalues. By using the presented basis set,
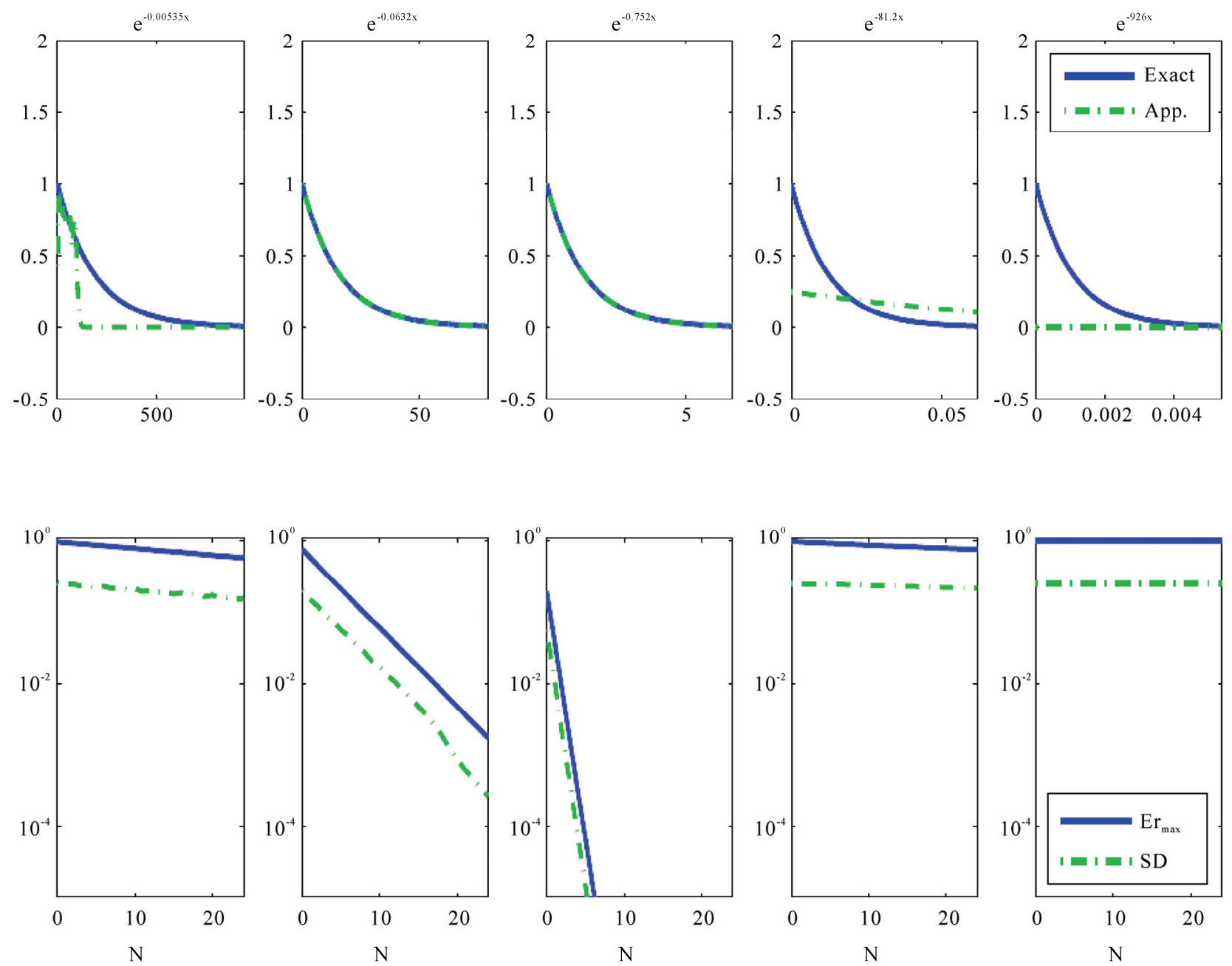

Figure 1. The approximations (top) and the maximum approximation errors and their standard deviations (bottom) of the five exponentially decaying functions using unscaled physical Laguerre basis set. The used decaying rates are $0.00535,0.0632$, 0.752, 81.2, and 926 . 

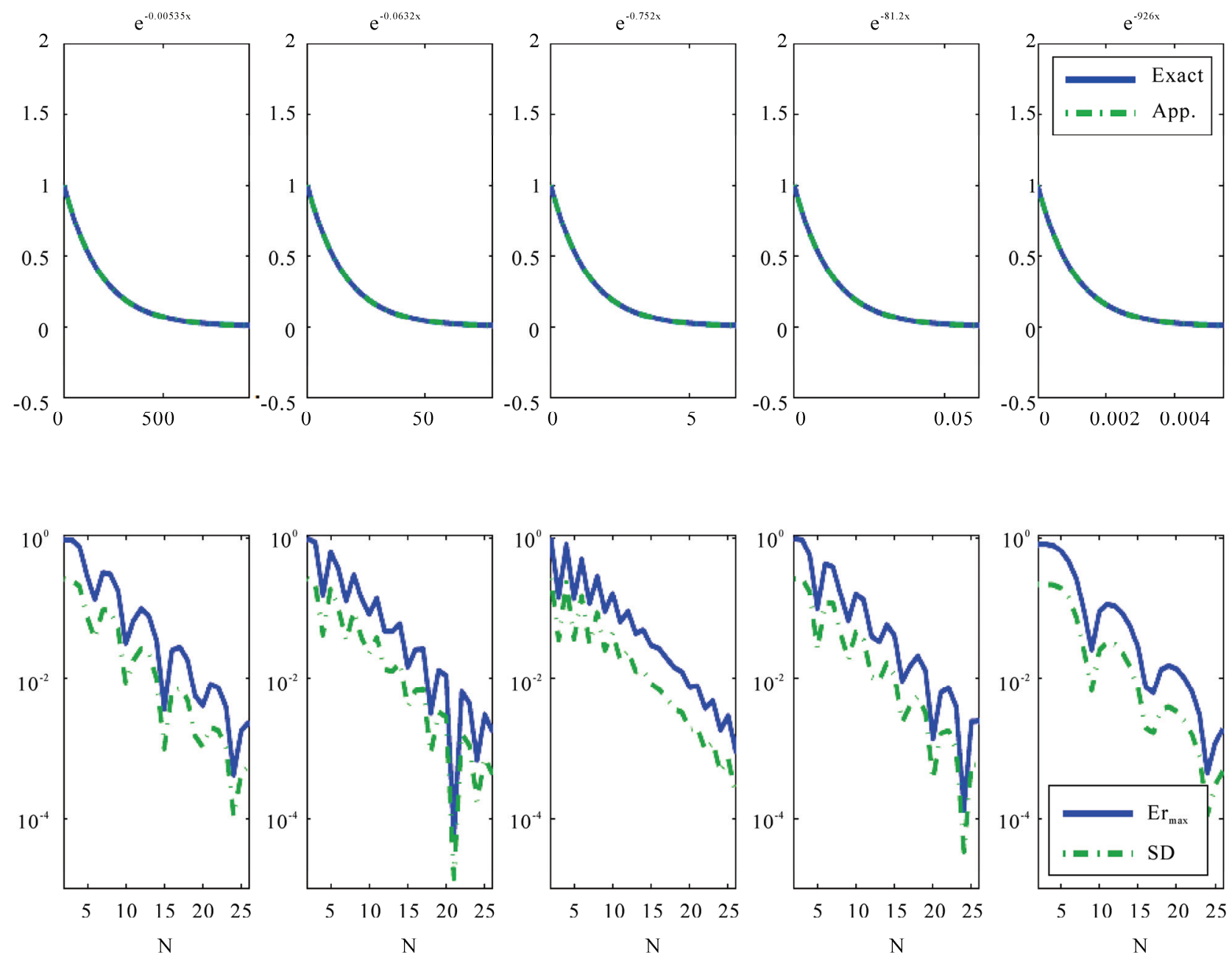

Figure 2. The approximations (top) and the maximum approximation errors and their standard deviations (bottom) of the five exponentially decaying functions using the presented basis set with the same bases. The used decaying rates are 0.00535 , $0.0632,0.752,81.2$, and 926 .

this can be done explicitly.

The presented set is used also to approximate wider spectrum of decaying rates using the same bases. The used decaying rates in this analysis are 1:35 $\times 10^{-6}, 2.32$ $\times 10^{-4}$, and $3: 2$, and 4120 , and 526000. Again, these were picked randomly. In this analysis, the used parameters are $b=10, d_{s}=-6$, and $d_{e}=6$. Figure 3 shows the obtained approximations and their associated errors. It is clear that the approximation is very efficient for all the functions even with this wider spectrum of decaying rates.

\subsection{Single Semiconductor QW without Biasing Field}

In this subsection, a semiconductor quantum well is analyzed by MDSM and using the presented basis set. Electronic states in QWs are described by the envelopes of the Bloch wave function. These are the solutions of the effective mass approximation of Schrodinger wave equation, which is:

$$
\frac{-\hbar^{2}}{2} \frac{d}{d x}\left(\frac{1}{m^{*}(x)} \frac{d \phi(x)}{d x}\right)+V(x) \phi(x)=\mathcal{E} \phi(x)
$$

where $\hbar$ is the normalized Planck constant, $m^{*}(x)$ is the effective mass, $V(x)$ is the $\mathrm{QW}$ potential in the growth direction, which is assumed to be the $x$-axis, $\mathcal{E}$ is the quantized energy level, and $\phi(x)$ is the quantized state. The studied structure is simply a thin layer of GaAs sandwiched in $\mathrm{Al}_{0.3} \mathrm{Ga}_{0.7}$ As. The width of the QW layer is $20 \mathrm{~nm}$. The structure is divided into three domains and the structural parameters and the selected expansion basis sets in each domain are shown in the Table 1. This structure can be analyzed analytically, where the energy states are the solutions of the following characteristic 

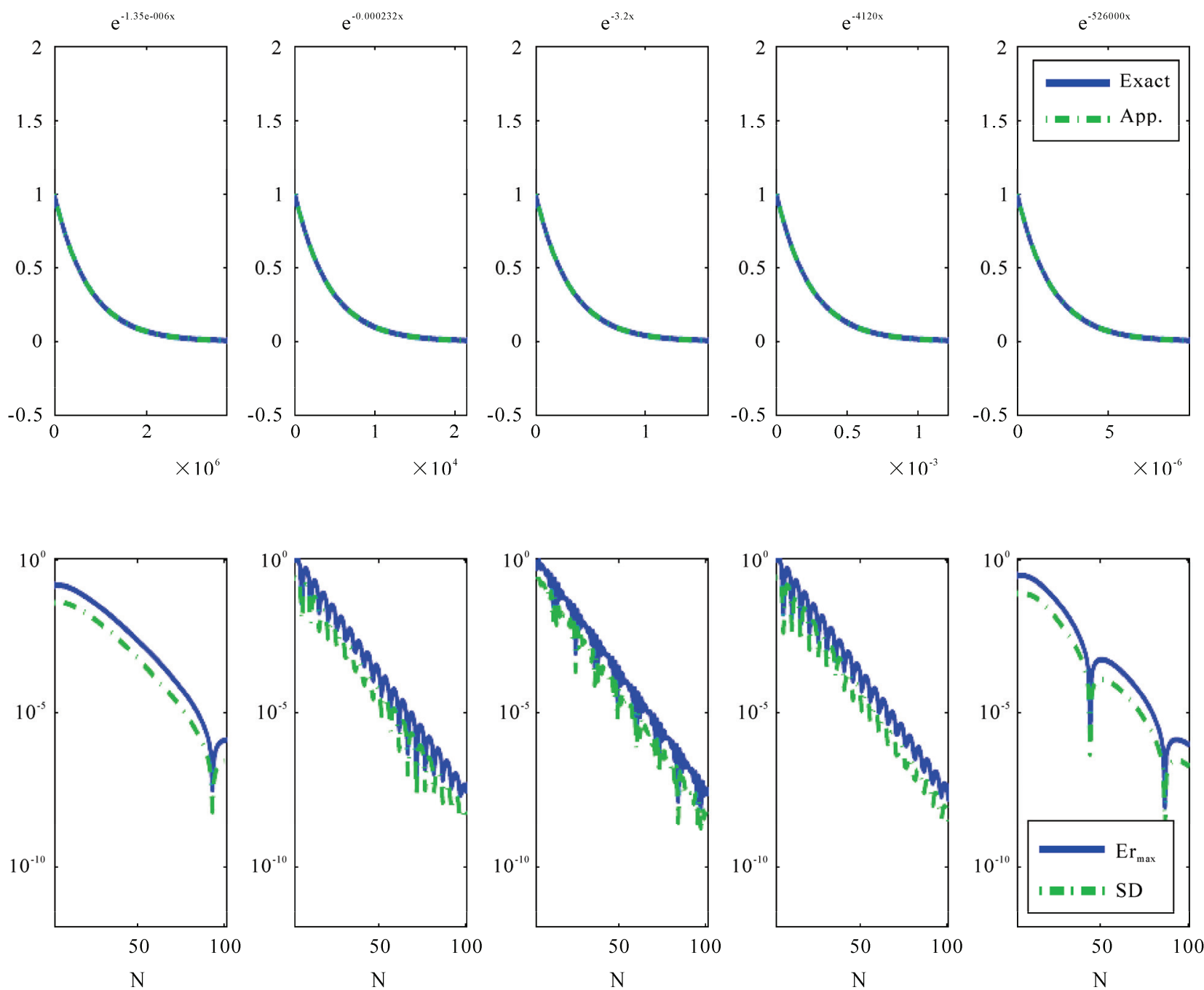

Figure 3. Approximations (top) and maximum approximation errors and their standard deviations (bottom) of the five exponentially decaying functions using the presented basis set with the same bases. The used decaying rates are $1.35 \times 10^{-6}, 2.32 \times$ $10^{-4}$, and 3.2, and 4120 , and 526000 .

equation:

$$
\left(1+\gamma^{2}\right) e^{i \delta L}-\left(1-\gamma^{2}\right) e^{-i \delta L}=0
$$

where

$$
\begin{gathered}
\delta=\sqrt{\mathcal{E} \frac{2 m_{w}}{\hbar^{2}}} \\
\beta=\sqrt{\left(V_{b}-\mathcal{E}\right) \frac{2 m_{b}}{\hbar^{2}}}
\end{gathered}
$$

and

$$
\gamma=i \frac{m_{b}}{m_{w}} \frac{\delta}{\beta}
$$

where $m_{b}$ and $m_{w}$ are the effective masses in the barrier and the well and $L$ is the width of the well. Table 2 shows the obtained results and compare them to the ana- lytical solutions and the results obtained recently by Huang using collocation spectral method (CSM) [11]. For these results, 15 basis are used for each domain. This is really overkilling in accuracy; but it is shown to reveal the accuracy of the presented method. The relative errors of the results obtained using the presented method and the exact solution are shown in Figure $\mathbf{4}$ against the number of the used basis in each domain. It is clear that acceptable results can be achieved with only 9 bases in each domain. In QWs, an accuracy tolerance of 0.001 $\mathrm{meV}$ is usually suffcient. The speed of the method mainly depends on the largest used matrix in the analysis. Since three domains used in this analysis and the used number of bases is the same on all of them (this is not necessary), the largest matrix size is $3 N \times 3 N$ where $N$ is the number of the used bases in each domain. We reach 
Table 1. The structural parameters of the studied QW.

\begin{tabular}{ccccc}
\hline & Interval (nm) & $m^{*}=m_{0}$ & $V(x)(\mathrm{meV})$ & Used basis set \\
\hline D1 & $(-\infty,-10)$ & 0.0919 & 225 & The presented exponential basis set \\
D2 & $(-10,10)$ & 0.067 & 0 & Chebyshev basis set (the first kind) \\
D3 & $(10, \infty)$ & 0.0919 & 225 & The presented exponential basis set \\
\hline
\end{tabular}

Table 2. Energy levels of the first studied QW in meV.

\begin{tabular}{cccc}
\hline Energy level & Exact & This work & CSM \\
\hline$E_{1}$ & 9.965713824282 & 9.965713824282 & 9.965616696787 \\
$E_{2}$ & 39.766000321692 & 39.7660000321691 & 39.765614440578 \\
$E_{3}$ & 88.920714571890 & 88.920714571958 & 88.9198632662562 \\
$E_{4}$ & 155.586554286161 & 155.586554286589 & 155.585132856243 \\
\hline
\end{tabular}

the machine accuracy with 15 basis in each domain where the largest matrix is only $45 \times 45$. This is handled very easily and rapidly. The whole analysis lasted about half a second. The envelopes of the four quantized states are shown in Figure 5.

\section{Conclusions}

A non-orthogonal basis set for half-bounded domain is presented and applied within the frame of MDSM. It is applied and compared with other methods and exhibits very high accuracy and geometric convergence. In MDSM, approximating exponentially decaying functions is one of the main problems mainly because of zero crossing and narrow-ranged decaying rates. The presented basis set overcomes this for real-valued functions.

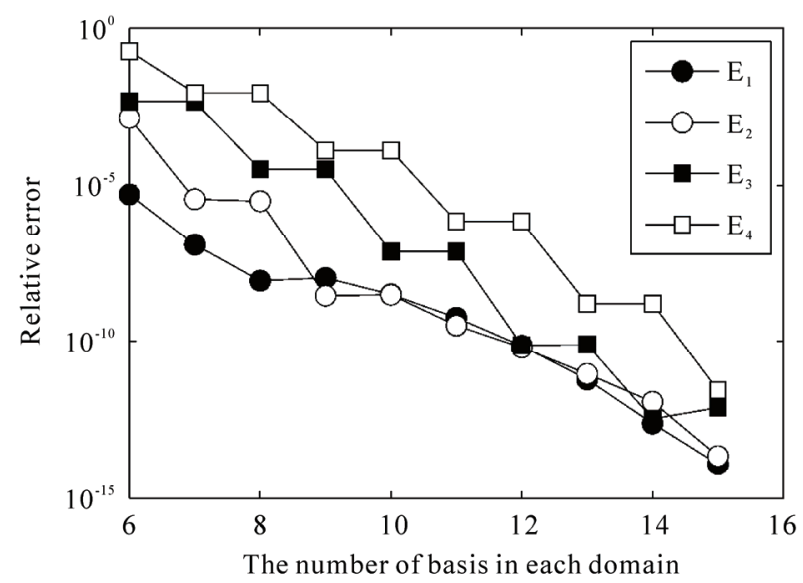

Figure 4. The relative error in energy levels between MDSM using the presented exponential set and the exact solutions of the studied QW.

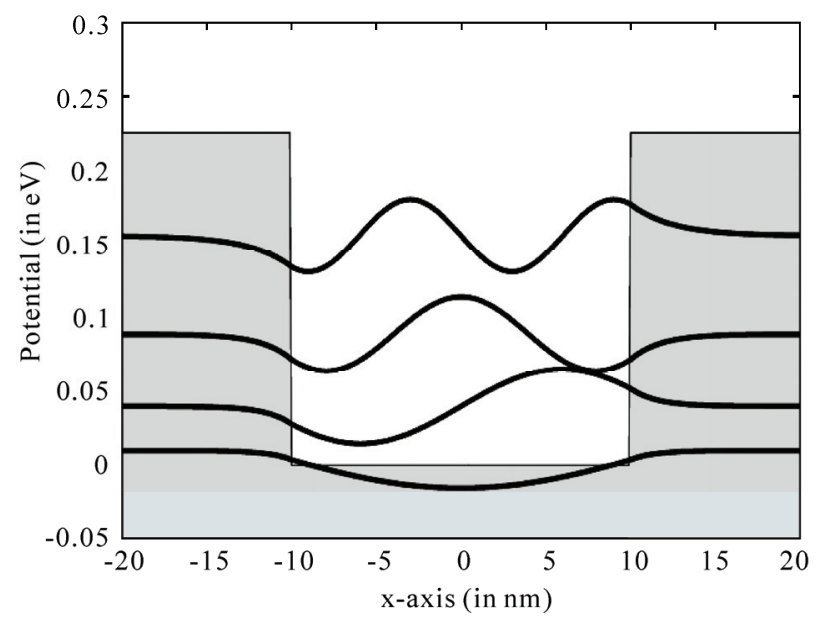

Figure 5. The envelopes of the four quantized states in the studied QW.

With the proper selection of parameters, it covers huge spectrum of decaying rates as shown.

\section{References}

[1] P. Grandclement and J. Novak, "Spectral Methods for Numerical Relativity," Living Reviews in Relativity, Vol. 12, No. 1, 2009, pp. 1-107.

[2] J. Boyd, "Chebyshev and Fourier Spectral Methods," Dover Publications, Mineola, 2001.

[3] C. G. Canuto, M. Y. Hussaini, A. M. Quarteroni and T. A. Zang, "Spectral Methods: Fundamentals in Single Domains," 1st Edition, Springer, New York, 2006.

[4] A. Toselli and O. Widlund, "Domain Decomposition Methods," Springer, Berlin, 2004.

[5] D. Fructus, D. Clamond, J. Grue and Â. Kristiansen, "An Efficient Model for Three-Dimensional Surface Wave 
Simulations: Part I: Free Space Problems," Journal of Computational Physics, Vol. 205, No. 2, 2005, pp. 665685.

[6] B.-Y. Guo and J. Shen, "Irrational Approximations and their Applications to Partial Differential Equations in Exterior Domains," Advances in Computational Mathematics, Vol. 28, No. 3, 2008, pp. 237-267.

[7] B.-Y. Guo, "Jacobi Spectral Approximations to Differential Equations on the Half Line," Journal of Computational Mathematics, Vol. 18, No. 1, 2000, pp. 95-112.

[8] J. Valenciano and M. Chaplain, "A Laguerre-Legendre Spectral-Element Method for the Solution of Partial Differential Equations on Infinite Domains: Application to the Diffusion of Tumour Angiogenesis Factors," Mathematical and Computer Modelling, Vol. 41, No. 2-3, 2005, pp. 1171-1192.

[9] V. Korostyshevskiy and T. Wanner, "A Hermite Spectral Method for the Computation of Homoclinic Orbits and Associated Functionals," Journal of Computational and Applied Mathematics, Vol. 206, No. 2, 2007, pp. 9861006.

[10] J. Shen and L.-L. Wang, "Some Recent Advances on Spectral Methods for Unbounded Domains," Communications in Computational Physics, Vol. 5, No. 2-4, 2009, pp. 195-241.

[11] C.-C. Huang, "Semiconductor Nanodevice Simulation by Multidomain Spectral Method with Chebyshev, Prolate Spheroidal and Laguerre Basis Functions," Computer Physics Communications, Vol. 180, No. 1, 2009, pp. 375 383. 